\title{
Haemolytic Anaemia Following High Dose Intravenous Immunoglobulin Treatment for Epidermolysis Bullosa Acquisita
}

\author{
Sarah Madeline Brown*, Philip Jeremy Hampton \\ Department of Dermatology, The Newcastle upon Tyne Hospitals NHS Foundation Trust, Royal Victoria \\ Infirmary, Newcastle, UK \\ Email: "sarahmadelinebrown@nhs.net
}

Received 11 October 2015; accepted 11 January 2016; published 14 January 2016

Copyright @ 2016 by authors and Scientific Research Publishing Inc.

This work is licensed under the Creative Commons Attribution International License (CC BY). http://creativecommons.org/licenses/by/4.0/

c) (i) Open Access

\begin{abstract}
Background: Epidermolysis bullosa aquisita (EBA) is a severe acquired blistering skin disease that is often resistant to prednisolone but can respond well to intravenous immunoglobulin infusion (IVIg). Main Observations: We describe the case of a 35 years old male patient with EBA who developed clinically significant haemolytic anaemia with a drop in $\mathrm{Hb}$ from $15.3 \mathrm{~g} / \mathrm{dL}$ to a nadir of $8.4 \mathrm{~g} / \mathrm{dL}$ within 5 days post IVIg infusion. The patient was blood group $\mathrm{A}$ and the IVIg batch was found to have a high titre of anti-A immunoglobulin. Conclusions: IVIg is an effective treatment for EBA. Haemolysis associated with IVIg has not previously been reported in the dermatology literature but review of data from other specialties shows that the problem is well recognised. Dermatologists using IVIg should be aware of this potential complication and patients should be consented appropriately and warned about this potential side effect.
\end{abstract}

\section{Keywords}

Haemolytic Anaemia, Haemolysis, Intravenous Immunoglobulin, Epidermolysis Bullosa Aquisita, EBA

\section{Introduction}

Epidermolysis bullosa acquisita (EBA) is a rare immunobullous disorder characterised by autoantibodies against collagen VII and the development of blisters which heal with scarring [1]. Treatment can be challenging and standard immunosuppressive regimes combining prednisolone plus azathioprine or mycophenolate mofetil are

${ }^{*}$ Corresponding author.

How to cite this paper: Brown, S.M. and Hampton, P.J. (2016) Haemolytic Anaemia Following High Dose Intravenous Immunoglobulin Treatment for Epidermolysis Bullosa Acquisita. Pharmacology \& Pharmacy, 7, 25-28.

http://dx.doi.org/10.4236/pp.2016.71004 
not always effective. Intravenous immunoglobulin (IVIg) is used as a third line agent in the management of EBA. We describe a case of a 35 years old male, blood group A patient with epidermolysis bullosa acquisita (EBA) who developed haemolytic anaemia following the administration of high dose intravenous immunoglobulin (IVIg).

\section{Case Report}

A 35 years old male presented with a 1 year history of a blistering eruption. He was otherwise well apart from a past history of vitiligo and at presentation was taking no medication. The distribution of blisters was highly variable with blisters developing at the oral mucosa, forehead, arms, hands and the genitals. Scarring had developed at the wrists (Figure 1). Indirect immunofluorescence on salt split skin showed linear IgG at the floor of the blister. A collagen VII ELISA was positive at a titre of 37 arbitrary units (upper limit of normal, 6 arbitrary units). This confirmed the diagnosis of epidermolysis bullosa acquisita (EBA).

Initial treatment over 2 years included immunosuppression with prednisolone plus azathioprine (150 mg daily), terminated following elevated ALT. He then received prednisolone plus mycophenolate mofetil ( 2 g per day). Over this 2-year period his cumulative prednisolone dose was $8810 \mathrm{mg}$ with a mean dose of $12.5 \mathrm{mg} / \mathrm{day}$. There were some short periods of disease remission but the blistering recurred, despite periods on higher doses of prednisolone. To regain disease control and to limit the cumulative dose of oral prednisolone, IVIg was selected as the next treatment. The first course of treatment was of IVIg, (Octagam) at a dose of 160 g over 4 days (500 mg/kg/day). There were no problems and the rate of new blisters decreased along with healing of eroded areas. Two months later a further infusion was planned. Due to a lack of availability of Octogam the next course of treatment was with a different brand of IVIg. The patient received $160 \mathrm{~g}$ of $10 \%$ IVIg (Privigen) in divided doses by slow IV infusion over 4 days (500 mg/kg per day). Two days following Privigen infusion he presented with symptoms of anaemia; with shortness of breath, clinically jaundiced and with skin mottling. On investigation his haemoglobin was found to have dropped from a baseline of $15.3 \mathrm{~g} / \mathrm{dL}$ to a nadir of $8.4 \mathrm{~g} / \mathrm{dL}$ within 5 days post IVIg infusion (Figure 2). Other biochemical markers of haemolysis included a raised bilirubin, raised $\mathrm{LDH}$ and a positive direct antiglobulin test. A blood film showed features consistent with haemolysis with spherocytes, polychromasia and a reticulocytosis.

A two unit blood transfusion was required. The episode of haemolysis was investigated by blood transfusion services and the Privigen bottle transfused was found to have a high titre of anti-A antibodies. Our patient was blood group A positive. The patient recovered quickly from the haemolysis and went on to receive further IVIg infusions with no evidence of haemolysis. Pre-infusion IVIg titres of anti-A1 IgG were checked in subsequent transfusions and given only when present at less than 1/64 dilution. The EBA blisters responded reasonably well
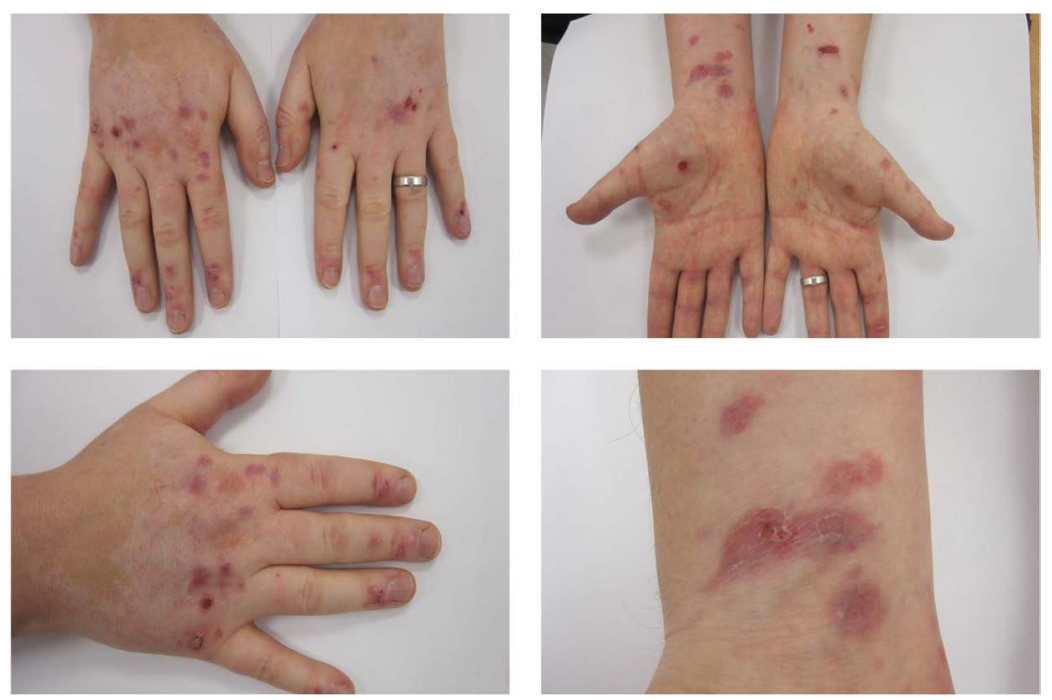

Figure 1. Clinical appearances of EBA blistering at the wrists and hands. Scarring has developed in some areas following sub basement membrane blistering secondary to anti-collagen VII antibody mediated inflammation. 


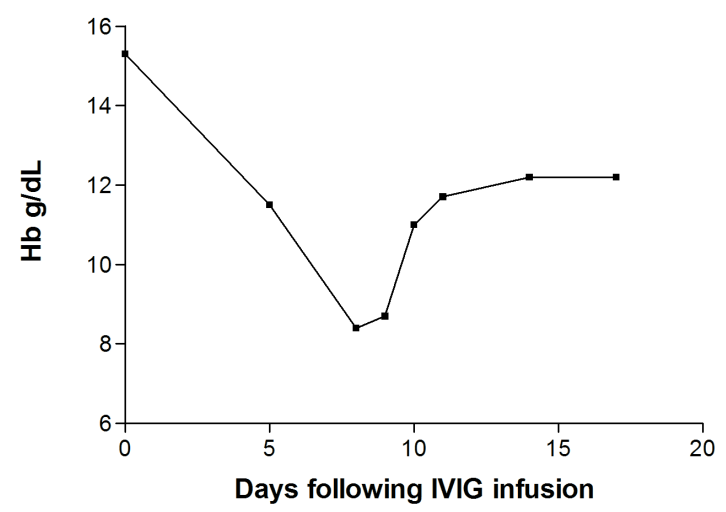

Figure 2. Levels of haemoglobin following IVIg. The haemoglobin concentration fell from $15.3 \mathrm{~g} / \mathrm{dL}$ to 8.4 g/dL over 8 days.

to IVIg and the disease went into remission with maintenance treatment of mycophenolate mofetil $500 \mathrm{mg}$ BD, dapsone $50 \mathrm{mg}$ OD and prednisolone $5 \mathrm{mg}$ OD. More recently, following a further relapse, the patient has received two infusions of rituximab $1000 \mathrm{mg}$ with a 14-day interval with good benefit and is well controlled with $7.5 \mathrm{mg}$ prednisolone only.

\section{Discussion}

Although there are no randomised controlled trials of IVIg in EBA, numerous case series and reports have demonstrated good results [2] [3]. The incidence of clinically significant haemolytic anaemia post IVIg is relatively rare [4]. This is the first reported case involving IVIg induced haemolysis when treating an immunobullous disease despite relatively widespread use of IVIg in the treatment of EBA and pemphigus. IVIg is prepared by collecting pooled immunoglobulin from blood donors and therefore the composition of different batches of IVIg does have some variation. The titres of anti-A and anti-B blood group antigens varies between different batches of IVIg. The problem has been more widely reported in the haematology literature and also in relation to patients with neurological diseases such as Guillain-Barré syndrome, multifocal motor neuropathy, chronic inflammatory demyelinating polyneuropathy, and dermatomyositis [5] [6]. The risk of haemolysis is greater in those patients receiving high dose IVIg (doses greater than or equal to $2 \mathrm{~g} / \mathrm{kg}$ [7]). The risk of haemolysis is also greater when the recipient has a non-O blood type with one review finding that $81 \%$ cases of post IVIg haemolysis were found in patients with blood group-A [7]. Another risk factor which can predispose to haemolysis is the use of IVIg brands with high titre anti-A/B IgG antibodies [7]. In one case study all identified cases of haemolysis involved the use of the IVIg products Gamunex, Gammagard liquid or Privigen [7]. The risk of haemolysis is highest in the high $\mathrm{A} / \mathrm{B}$ titre isosmolar preparations, however the lower titre preparations are usually hyperosmotic and have a greater risk of causing acute renal failure or thrombosis [7]. One case series identified 16 cases of IVIg induced haemolysis out of approximately 1000 patients receiving IVIg over a 2.5-year period. It was identified that haemolysis occurred within 12 hours to 10 days following IVIg administration, with the lowest level of haemoglobin identified between 1 day and 2 weeks post infusion [8]. Mild cases of clinically insignificant IVIg induced haemolysis may occur relatively often in blood group A and B patients. Post infusion full blood count in clinically healthy patients is not carried out in all hospitals.

IVIg-related haemolysis has been demonstrated to involve anti-A and anti-B haemagglutinin in the IVIg preparations as well as both IgG and complement mediated hemolysis. It has been proposed that the haemolysis takes place in two stages or as a two hit process [8]. The first hit is felt to be related to passive transfer of anti-A and anti-B haemagglutinin. These haemagglutinins cause red cell aggregation. The second hit is related to the increased degradation and loss of sensitised red blood cells in an individual with an underlying inflammatory state due to erythrophagocytosis in the presence of complement. High dose IVIg fixes complement onto red cells resulting in haemolysis and also binds to red bloods cells which are then removed by the spleen [7] [9]. The current European Pharmacopoeia have suggested guidelines such that the titre of anti-A and anti-B haemagglutinins should not be detected at the 1:64 dilution, however there are reports in literature of haemolysis occurring regardless of these limits [7] [9]. 


\section{Conclusion}

EBA can be a difficult disease to control and IVIg is a useful treatment option. We report the first described case of clinically significant IVIg induced haemolysis in a patient with EBA. Dermatologists need to be aware of the possibility of haemolysis and vigilance is important as patients can become symptomatic requiring prompt assessment and monitoring. A rational approach to monitoring for post IVIg haemolytic anaemia would be to monitor full blood count and bilirubin 24 to 48 hours post infusion and again 1 week later. Patients must be fully informed and consented regarding this potential complication. In order to reduce the risk of occurrence of IVIg associated haemolysis, pre-infusion isohaemagglutinin titres can be measured and given only when present at less than 1/64 dilution (European Pharmacopeia Recommendation). Alternatively a direct antiglobulin test between IVIg product and recipients blood could be performed [7]. These are especially relevant in patients with non-O blood groups. Since most patients receive IVIg without complication the need for these extra tests as part of routine management would need to be discussed with the patient. In many cases blood monitoring will be adequate.

\section{Conflict of Interest}

None of the authors have any conflicts of interest to declare.

\section{References}

[1] Kim, J.H., Kim, Y.H., et al. (2013) Serum Levels of Anti-Type VII Collagen Antibodies Detected by Enzyme-Linked Immunosorbent Assay in Patients with Epidermolysis Bullosa Acquista Are Correlated with the Severity of Skin Lesions. Journal of the European Academy of Dermatology and Venereology, 27, e224-e230. http://dx.doi.org/10.1111/j.1468-3083.2012.04617.x

[2] Intong, L.R. and Murrell, D.F. (2011) Management of Epidermolysis Bullosa Acquista. Dermatologic Clinics, 29, 643647. http://dx.doi.org/10.1016/j.det.2011.06.020

[3] Czernik, A., Toosi, S., et al. (2012) Intravenous Immunoglobulin in the Treatment of Autoimmune Bullous Dermatoses: An Update. Autoimmunity, 45, 111-118. http://dx.doi.org/10.3109/08916934.2011.606452

[4] Berard, R., Whittemore, B., et al. (2012) Hemolytic Anaemia Following Intravenous Immunoglobulin Therapy in Patients Treated for Kawasaki Disease: A Report of 4 Cases. Pediatric Rheumatology Online Journal, 10, 10. http://dx.doi.org/10.1186/1546-0096-10-10

[5] Hughes, R.A., Dalakas, M.C., et al. (2009) Clinical Applications of Intravenous Immunoglobulins in Neurology. Clinical \& Experimental Immunology, 158, 34-42. http://dx.doi.org/10.1111/j.1365-2249.2009.04025.x

[6] Markvardsen, L.H., Christiansen, I., et al. (2014) Hemolytic Anaemia Following High Dose Intravenous Immunoglobulin in Patients with Chronic Neurological Disorders. European Journal of Neurology, 21, 147-152. http://dx.doi.org/10.1111/ene.12287

[7] Kahwaji, J., Barker, E., et al. (2009) Acute Hemolysis after High-Dose Intravenous Immunoglobulin Therapy in Highly HLA Sensitized Patients. Clinical Journal of the American Society of Nephrology, 4, 1993-1997. http://dx.doi.org/10.2215/CJN.04540709

[8] Daw, Z., Padmore, R., et al. (2008) Hemolytic Transfusion Reactions after Administration of Intravenous Immune (Gamma) Globulin: A Case Series Analysis. Transfusion, 48, 1598-1601. http://dx.doi.org/10.1111/j.1537-2995.2008.01721.x

[9] Padmore, R.F. (2012) Hemolysis upon Intravenous Immunoglobulin Transfusion. Transfusion and Apheresis Science, 46, 93-96. http://dx.doi.org/10.1016/j.transci.2011.11.004 